\title{
Template Matching for Profile Measurement Based on Levenberg-Marquardt Algorithm
}

\author{
Qingxia Xu, Xiaodong Chai, Shubin Zheng, Wenfa Zhu, Lei Zhang \\ Rail Transportation College, Shanghai University of Engineering Science, Shanghai, China \\ Email: xuqingxia_cool@163.com
}

Received 20 May 2014; revised 16 June 2014; accepted 12 July 2014

Copyright (C) 2014 by authors and Scientific Research Publishing Inc.

This work is licensed under the Creative Commons Attribution International License (CC BY). http://creativecommons.org/licenses/by/4.0/

c) (i) Open Access

\begin{abstract}
This paper proposes the Levenberg-Marquardt algorithm to solve the problem of a big error while matching the measurement profile with the template profile. In order to achieve more accurate matching result, error function of the two profiles should be structured firstly. And the next step is optimizing the parameters of transform matrix of error function. To be specific, the error is about the corresponding point distance, and optimization parameters are the rotation variables and the translation variables of the transformation matrix. The experimental result of the system shows that using the method of template matching for profile measurement based on Levenberg-Marquardt algorithm is feasible.
\end{abstract}

\section{Keywords}

Template Matching, Levenberg-Marquardt Algorithm, Error Function, Parameter Optimization

\section{Introduction}

At present, one of the common methods about template matching has been used neural network to classify the training to finish matching [1]. Another method is depending on the information of the area overlapping rate and the center relative movement rate from image sequences, to complete the matching for object recognition [2] [3]. These methods have their own characters. To get a more effective method, Levenberg-Marquardt algorithm is presented. This algorithm has both fast convergence properties of Gauss Newton method and global search properties of the gradient search method. The L-M algorithm optimizes the parameters of the initial contour transformation matrix, which can reduce the error between the measurement contour and the template contour, achieving more accurate matching. Transformation matrix is the matrix of changing the coordinate system of the original profile image into that of the template profile image.

Fast and accurate template matching method has a great significance in many areas. 


\section{The Principle of L-M Algorithm}

Now, the principle of L-M algorithm is introduced simply.

Error function is showed in Equation (1):

$$
F(x)=\frac{1}{2} \sum_{i=1}^{N} f_{i}^{2}(x)
$$

where $f_{i}(x)$ denotes the current error.

$x^{k}$ is variable value of $f_{i}(x)$ after iterating $k$ times, $x^{k+1}$ is the next step of iteration.

$$
x^{k+1}=x^{k}+\Delta^{k}
$$

The equation of the improved Gauss-Newton method:

$$
\Delta^{k}=-\left[J^{\mathrm{T}}\left(x^{k}\right) J\left(x^{k}\right)+\mu I\right]^{-1} J^{\mathrm{T}}\left(x^{k}\right) f\left(x^{k}\right)
$$

where $J(x)$ is Jacobi matrix of $F(x), I$ is unit matrix, $\mu$ is known as the proportional factor and $\mu>0$. When $\mu=0$ is true, Equation (3) is called Gauss-Newton method; when the value of $\mu$ is large, Equation (3) is similar to the gradient descent method. Iterating step by step, if it is closer to the optimization goal, $\mu$ is gradually decreased, with the rapid convergence of Gauss Newton method. On the contrary, if it is away from the optimization goal, $\mu$ is increasing gradually with the gradient descent method. Therefore, the L-M algorithm is a combination of Gauss Newton method and gradient descent method, with the advantages of both.

\section{Optimization of Parameters [4] [5]}

The contour matching is a process to obtain the best matching, using the same object obtained from different camera contour to transform into the same coordinate system. There is overlap part in two profile images, with the measurement contour image as the optimal image and the template contour image as the target image. The coordinate of optimal image can obtain the coordinate of target image through a transformation matrix. As usual, the transformation matrix has three rows and three columns, containing the rotation variables and the translation variables. The transformation matrix is denoted $H$ :

$$
H=\left(\begin{array}{lll}
h_{1} & h_{2} & h_{3} \\
h_{4} & h_{5} & h_{6} \\
h_{7} & h_{8} & h_{9}
\end{array}\right)
$$

where $h_{1}, h_{2}, h_{4}$ and $h_{5}$ represent the rotation variables, $h_{3}, h_{6}$ represent the translation variables. $h_{7}, h_{8}$ is very small usually, so they could be equal $0 . h_{9}$ is the normalized constant, so $h_{9}$ is considered to be 1 .Thus, there are six parameters to be optimized.

The key issue of template matching is how to reduce the matching error, and the paper utilizes the L-M algorithm to solve this problem. At first, a certain number of corresponding feature points is determined, feature points from calibration. Then, the initial transformation matrix is obtained by calibration method.

Assuming the number of feature points is $n$. $\left(x_{i}, y_{i}\right)$ denotes the coordinate of original contour image. $\left(X_{i}, Y_{i}\right)$ is the coordinate after transforming by $\left(x_{i}, y_{i}\right)$, or known as measurement contour image. $\left(X_{i}^{\prime}, Y_{i}^{\prime}\right)$ represents he coordinate of template contour image. Because there are six parameters to optimize, the condition should be met in theory that $n \geq 3$ is true. However, $n$ is required to be much bigger than 3 for a better transformation matrix.

The relationship of $\left(x_{i}, y_{i}\right)$ and $\left(X_{i}, Y_{i}\right)$ is showed below:

$$
\left(\begin{array}{c}
X_{i} \\
Y_{i} \\
1
\end{array}\right)=\left(\begin{array}{lll}
h_{1} & h_{2} & h_{3} \\
h_{4} & h_{5} & h_{6} \\
h_{7} & h_{8} & h_{9}
\end{array}\right)\left(\begin{array}{c}
x_{i} \\
y_{i} \\
1
\end{array}\right)
$$

Error function is expressed concretely as:

$$
F(H)=\frac{1}{2} \sum_{i=1}^{n}\left[\left(Y_{i}^{\prime}-Y_{i}\right)^{2}+\left(X_{i}^{\prime}-X_{i}\right)^{2}\right]=\sum_{i=1}^{n} f_{i}^{2}(H)
$$


The differential on $f_{i}^{2}(H)$ in Equation (6):

$$
2 f_{i}(H) \mathrm{d} f_{i}(H)=2\left(Y_{i}^{\prime}-Y_{i}\right) \mathrm{d} Y_{i}+2\left(X_{i}^{\prime}-X_{i}\right) \mathrm{d} X_{i}
$$

With the addition, the deformation Equation of Equation (5):

$$
\begin{aligned}
& X_{i}=h_{1} x_{i}+h_{2} y_{i}+h_{3} \\
& Y_{i}=h_{4} x_{i}+h_{5} y_{i}+h_{6}
\end{aligned}
$$

Combine Equation (7) with Equation (8), Jacobi matrix can be solved.

$$
J(H)=\left|\begin{array}{cccc}
\frac{\partial f_{1}(H)}{\partial h_{1}} & \frac{\partial f_{1}(H)}{\partial h_{2}} & \cdots & \frac{\partial f_{1}(H)}{\partial h_{n}} \\
\frac{\partial f_{2}(H)}{\partial h_{1}} & \frac{\partial f_{2}(H)}{\partial h_{2}} & \cdots & \frac{\partial f_{2}(H)}{\partial h_{n}} \\
\vdots & \vdots & \ddots & \vdots \\
\frac{\partial f_{n}(H)}{\partial h_{1}} & \frac{\partial f_{n}(H)}{\partial h_{2}} & \cdots & \frac{\partial f_{n}(H)}{\partial h_{n}}
\end{array}\right|
$$

The calculation steps of L-M algorithm, as follows:

1) Given several conditions, one is $\varepsilon=10^{-6}$, another is $\lambda^{0}=10^{-3}$. $\varepsilon$ denotes the control constant for iterations being stopped. The initial vector $H^{k}(k=0)$ can be worked out by feature points.

2) The measurement contour image would be calculated out by the transformation matrix $\left(H^{k}\right)$, and also error function.

3) According to the former introduction, the next goal is to calculate Jacobi matrix.

4) Structure equation:

$$
\bar{N}_{k} \Delta^{k}=J\left(H^{k}\right) f\left(H^{k}\right)
$$

where $\bar{N}_{k}=J^{\mathrm{T}}\left(H^{k}\right) J\left(H^{k}\right)+\lambda^{k} I$.

5) Work out $\Delta^{k}$ according to the equation of the last step. If $\left\|\Delta^{k}\right\|<\varepsilon$ is true, stops iterating and puts out the results. Or, assume that $H^{k+1}=H^{k}+\Delta^{k}$ and calculate $F\left(X^{k+1}\right)$, and then make the following judgments:

a) If $F\left(X^{k+1}\right)<F\left(X^{k}\right)$, then $\lambda^{k+1}=\lambda / 10$ and $k=k+1$, turn to (2).

b) If $F\left(X^{k+1}\right)<F\left(X^{k}\right)$, then $\lambda^{k+1}=10 \lambda^{k}$, turn to (4).

\section{Experimental Results and Analysis}

The first step of the experiment is to obtain the rail profile. Figure 1 shows the experiment principle diagram. There are two cameras to be used, one is over the rail, and another is on the left. The images from the two cameras are cross, because there is the same part in the range of camera's vision. For the errors, intersection part does not fully coincide. The L-M algorithm is to reduce the error and make the coincidence degree of intersection part better. While each camera is on different coordinate system, the two coordinate system need to unify, Camera 1 and Camera 2 take pictures respectively, and the pictures are shown in Figure 2.

To unify the two coordinate systems is not hard, and it can be come true that rail change into calibration block. The initial matrix $\mathrm{H}$ has been worked out by feature points. Once coordinate systems are unified, rail measurement profile is shown in Figure 3. In Figure 2, the red line represents the template contour image, and the blue line represents the measured profile image to be optimized. The overlap of the template contour and profile measurement is magnified, as shown in Figure 4, which can clearly reflect the error is large, so using optimization algorithms to reduce the matching error is necessary. Figure 5 shows the experimental results.

Two groups' coordinate have been obtained, and then the error of the two groups' coordinate also can be solved. Original results are compared with results by using L-M algorithm in four aspects, including mean, standard deviation, maximum, and the numbers of value larger than 0.2 . The results are listed in Table 1 .

From Table 1, it is intuitively showed that the results of the L-M algorithm in the four indexes are better than the initial ones, so this method works. 


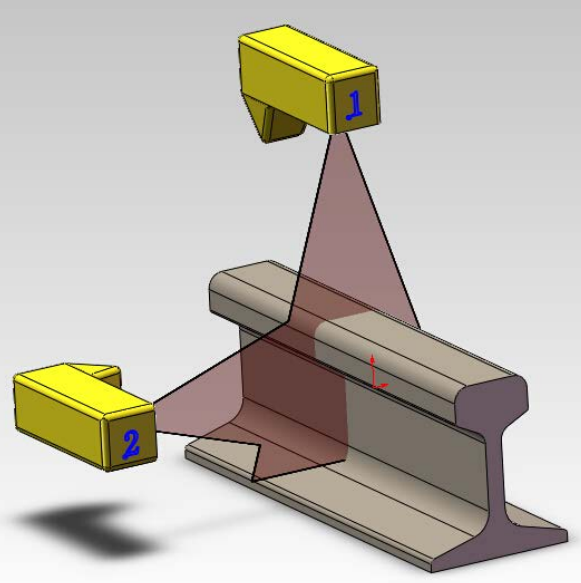

Figure 1. The experiment principle diagram.

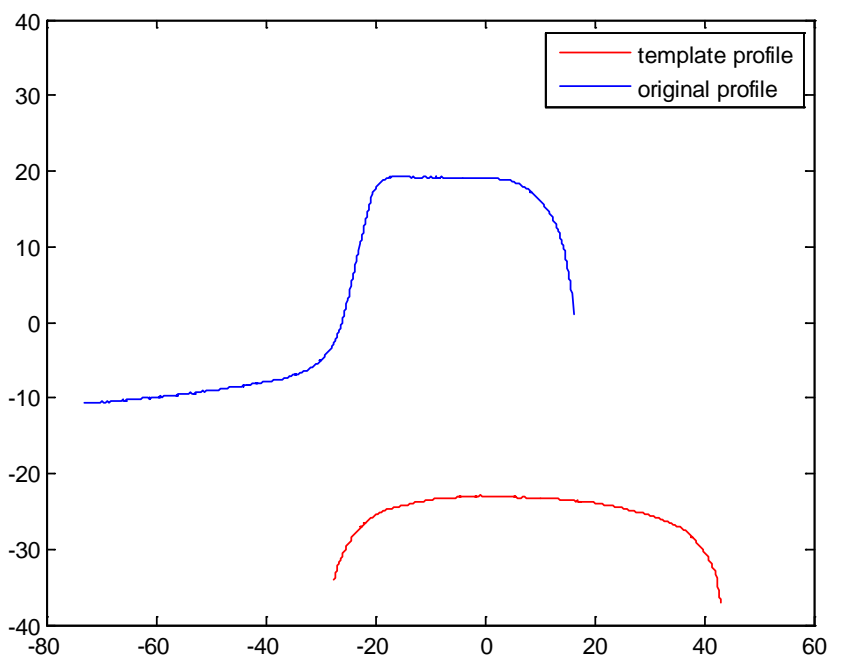

Figure 2. The picture from two cameras. (The red line from camera 1, the blue from camera 2).

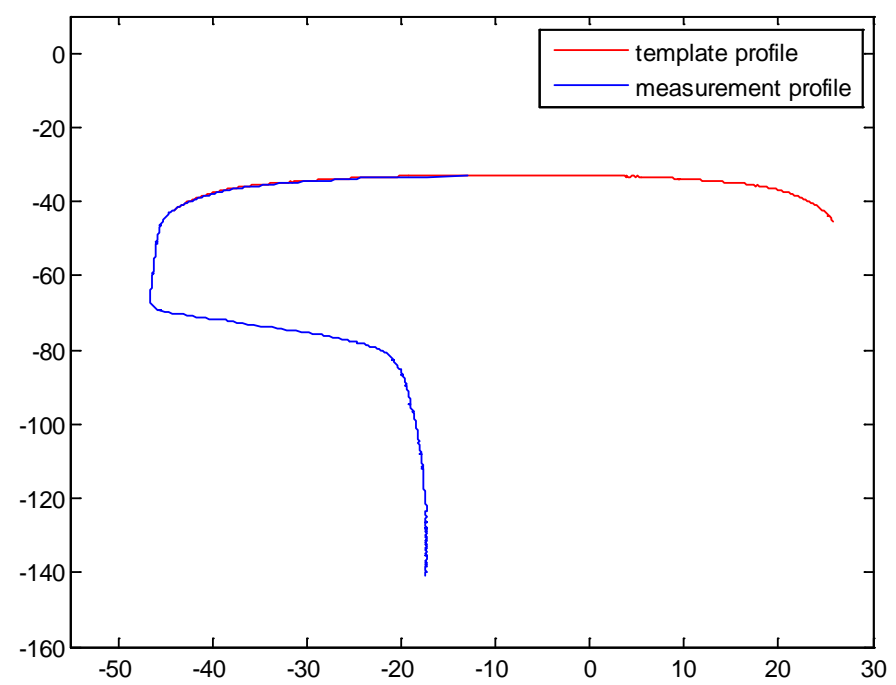

Figure 3. Rail profile unified on the same coordinate systems. 


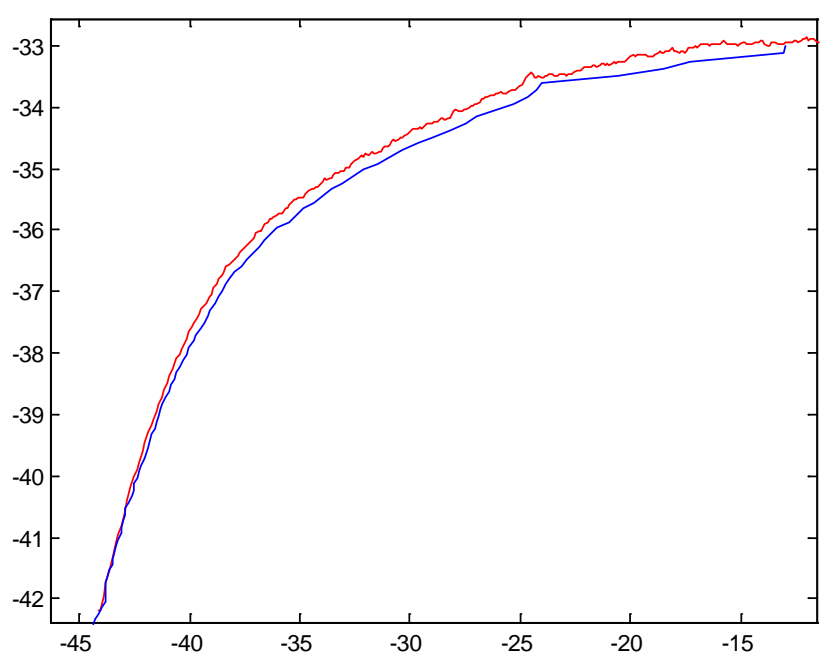

Figure 4. Overlap enlargement diagram.

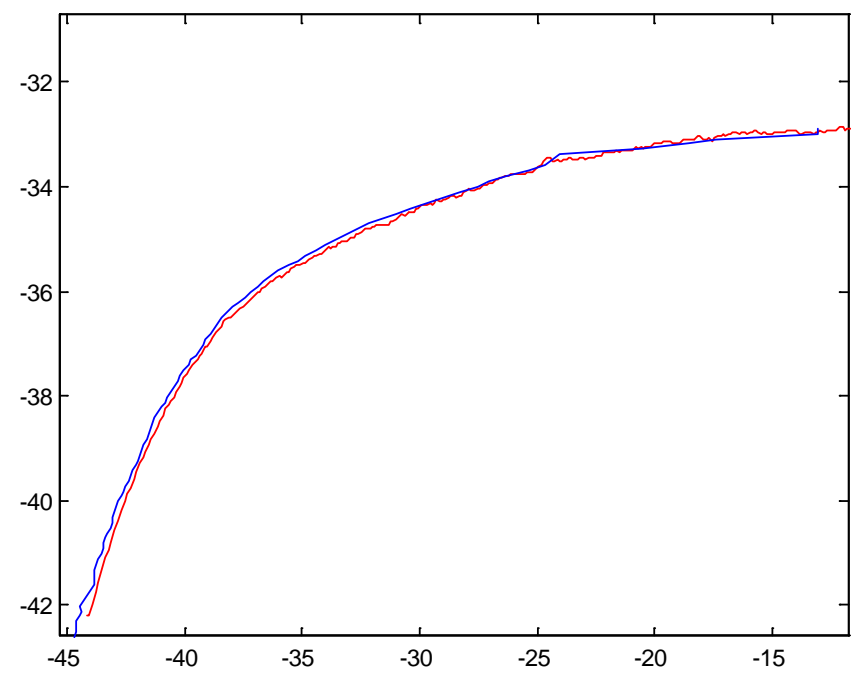

Figure 5. Matching result diagram by L-M algorithm.

Table 1. The comparison between Original results and results by using L-M algorithm.

\begin{tabular}{ccccc}
\hline item & Mean $(\mathrm{mm})$ & standard deviation $(\mathrm{mm})$ & Maximum $(\mathrm{mm})$ & $\begin{array}{c}\text { the numbers of value } \\
\text { larger than } 0.2\end{array}$ \\
\hline Origination & 0.1727 & 0.0637 & 0.3022 & 39 \\
L-M algorithm & 0.1516 & 0.0557 & 0.2848 & 14 \\
\hline
\end{tabular}

\section{Conclusion}

This paper proposes the L-M algorithm to optimize the parameters of the transformation matrix. This method can reduce the error between the measurement contour and the template contour. Experimental results show that the L-M algorithm can effectively solve the problem of the contour template matching.

\section{Acknowledgements}

Authors are grateful for the support of Scientific Research Innovation Project of Shanghai Education Commission (Granted No. 12YZ149, No. 12ZZ184) and Discipline Construction Project for Transportation Engineering (Granted No.: 13SC002), as well as Postgraduate Research Innovation Project (Granted No.: A-0903-13-01124). 


\section{References}

[1] Sun, Y., Zhou, G.H., Zhao, L.C. and Shi, P.F. (2000) Fast Template Matching Algorithm Based on the Projection. Journal of Shanghai Jiao Tong University, 34, 702-704.

[2] Yu, Z.X., Chen, L., Chen, H.P. and Zhang, X.L. (2013) A Method Integrated Multi-Feature for Recognizing Fire Based on Video Image Analysis. Industrial Control Computer, 26, 85-87.

[3] Fu, Y.-J., Yang, K.-T., Zou, W.-D. and He, X.-D. (2007) Image Mosaic Based on Levenberg-Marquardt Algorithm. Laser Journal, 28, 46-48.

[4] Wu, C.-F. (2008) Mathematical Methods in Computer Vision. Science Press, Beijing.

[5] Madsen, K., Nielsen, H.B. and Tingleff, O. (2004) Methods for Non-Linear Least Squares Problems. Informatics and Mathematical Modelling Technical University of Denmark, Lyngby. 
Scientific Research Publishing (SCIRP) is one of the largest Open Access journal publishers. It is currently publishing more than 200 open access, online, peer-reviewed journals covering a wide range of academic disciplines. SCIRP serves the worldwide academic communities and contributes to the progress and application of science with its publication.

Other selected journals from SCIRP are listed as below. Submit your manuscript to us via either submit@scirp.org or Online Submission Portal.
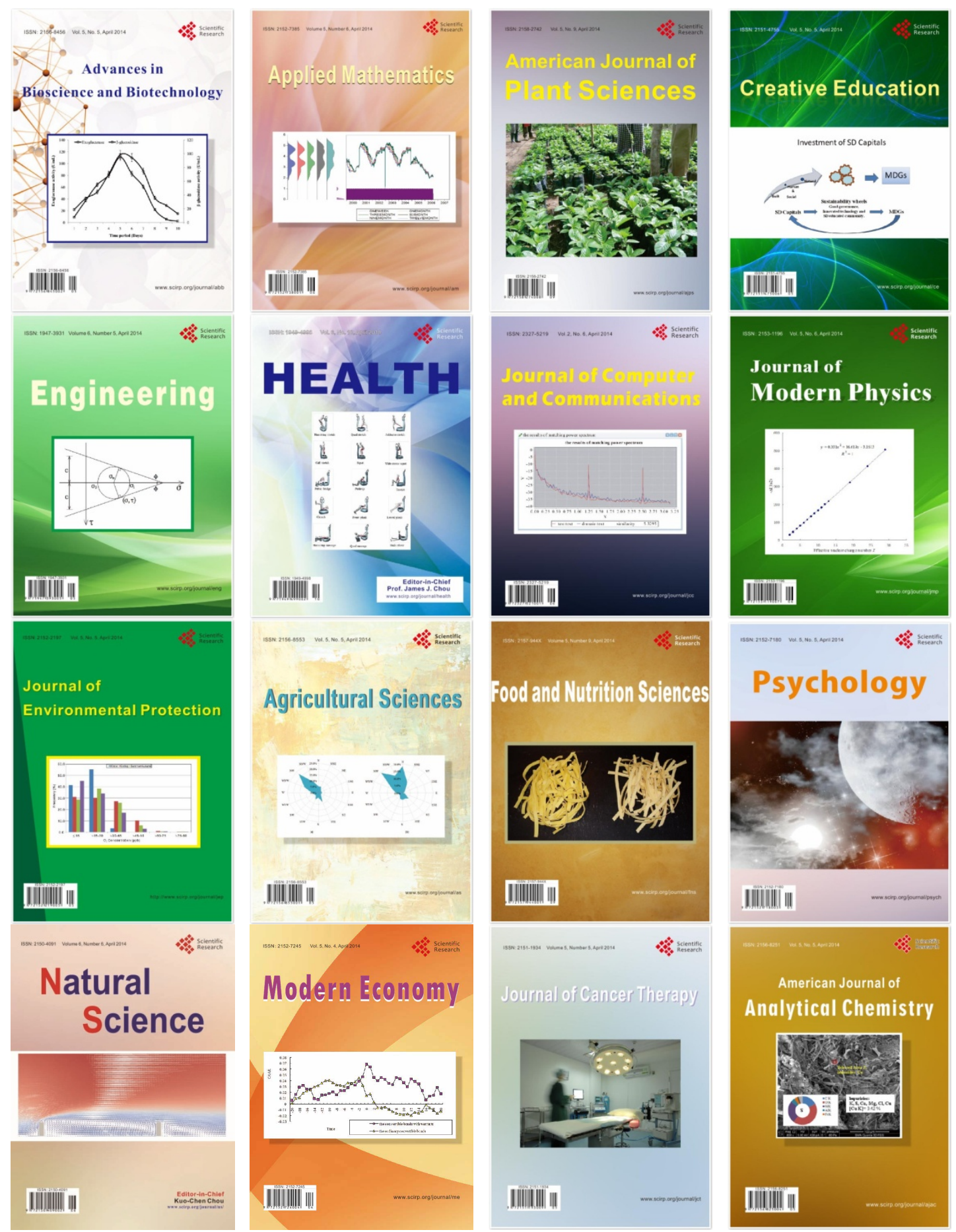